\title{
Evaluación de pacientes quirúrgicos no cardiacos mediante la calculadora ACS-NSIQP en la predicción de riesgo de neumonía, cardiaco, y tromboembolismo venoso
}

\author{
Assessment of non-cardiac surgical patients using \\ the ACS-NSQIP calculator to predict the risk of \\ pneumonia, cardiac complications and venous \\ thromboembolism
}

Indira Pachano-García, Jairo Rojano-Rada, Oswaldo Rodríguez-Arbeláez

- Caracas (Venezuela)

DOI: https://doi.org/10.36104/amc.2021.1853

\section{Resumen}

Objetivo: evaluación de pacientes quirúrgicos no cardiacos mediante la calculadora ACS-NSIQP para en la predicción de riesgo de neumonía, cardiaco, y tromboembolismo venoso dentro de los primeros 30 días del posoperatorio en pacientes con cirugía no cardiaca.

Métodos: se realizó un estudio de único centro, transversal y correlacional en pacientes a cargo de los servicios quirúrgicos del Hospital del Instituto Venezolano de los Seguros Sociales (IVSS) "Dr. Miguel Pérez Carreño", aplicando la calculadora ACS-NSQIP y las escalas estandarizadas a comparar índice de LEE, ACP y Caprini antes de la cirugía, posteriormente seguimiento vía telefónica enfocado en las respectivas complicaciones posoperatorias a los 15 y 30 días posoperatorios.

Resultados: del total de 98 pacientes que tuvieron seguimiento la edad media fue de $55 \pm 14$, correspondiendo $53(54.1 \%)$ al sexo femenino. En general hubo 23 complicaciones en $22.4 \%$ de la población; con más frecuencia neumonía en 14 sujetos $(14.2 \%)$, seguido por siete (7.2\%) cardiovascular y dos (2.02\%) tromboembolismo venoso. La estimación de riesgo para neumonía fue de AUC 0.695 ( $\mathrm{p}=0.017$ ) y cardiacas de AUC 0.739 ( $\mathrm{p}=0,036)$, evidenciando una asociación significativa para el evento correspondiente.

Conclusión: la calculadora de riesgo universal ACS-NSQIP tiene utilidad aceptable, para predecir complicaciones posoperatorias como neumonía y eventos cardiovasculares. (Acta Med Colomb 2021; 46. DOI: https://doi.org/10.36104/amc.2021.1853).

Palabras clave: evaluación de riesgo, cuidado preoperatorio, neumonía, enfermedad cardiovascular, tromboembolismo venoso.

\footnotetext{
Abstract

Objective: to evaluate non-cardiac surgical patients using the ACS-NSQIP to predict the risk of pneumonia, cardiac complications and venous thromboembolism within the first 30 postoperative days in patients undergoing non-cardiac surgery.

Methods: this was a single-center, cross-sectional, correlational study in patients treated by the surgery department of the Hospital del Instituto Venezolano de los Seguros Sociales (IVSS) "Dr. Miguel Pérez Carreño", using the ACS-NSQIP calculator and standardized scales to compare the Lee, ACP and Caprini indices before surgery and during telephone follow up focused on the respective postoperative complications 15 and 30 days after surgery.

Results: of the 98 total patients who were followed, the mean age was $55 \pm 14$, and $53(54.1 \%)$ were female. Overall, there were 23 complications in $22.4 \%$ of the population; pneumonia was the most frequent with 14 subjects $(14.2 \%)$, followed by seven $(7.2 \%)$ with cardiovascular complica-
}

Dra. Indhira Pachano-García: Miembro Centro Biomédico de Investigación del Postgrado de Medicina Interna (CEBIMI); Dr. Jairo Rojano Rada: Director del Centro Biomédico de Investigación del Postgrado de Medicina Interna (CEBIMI); Dr. Oswaldo Rodríguez-Arbeláez: Jefe del Grupo de Preoperatoria e Interconsulta del Centro Biomédico de Investigación del Postgrado de Medicina Interna (CEBIMI). Universidad Central de Venezuela, Sede Hospital Central del Instituto Venezolano de los Seguros Sociales "Dr. Miguel Pérez Carreño". Caracas (Venezuela).

Correspondencia: Dr. Jairo Rojano-Rada. Caracas (Venezuela).

E-mail: cebimehmpc@gmail.com

Recibido: 20/IV/2020 Aceptado: 1/XII/2020 
tions and two $(2.02 \%)$ with venous thromboembolism. The estimated risk for pneumonia was AUC $0.695(\mathrm{p}=0.017)$, and for cardiac complications was AUC $0.739(\mathrm{p}=0.036)$, showing a significant association with the respective event.

Conclusion: the universal ACS-NSQIP risk calculator has acceptable utility for predicting post-operative complications such as pneumonia and cardiovascular events. (Acta Med Colomb 2021;

46. DOI: https://doi.org/10.36104/amc.2021.1853).

Key words: risk assessment, preoperative care, pneumonia, cardiovascular disease, venous thromboembolism

\section{Introducción}

En las últimas décadas, la población mundial ha experimentado un incremento en la prevalencia de enfermedades crónicas no transmisibles (ECNT) (1), como la obesidad, la hipertensión, la ateroesclerosis y diabetes mellitus tipo 2; calculándose 15 millones de muertes por año en personas entre 30 y 69 años, este hecho como consecuencia de la urbanización rápida y no planificada, la mundialización de modos de vida poco saludables y el envejecimiento de la población aumentando la prevalencia de enfermedades cardiovasculares (ECV), representando así en la actualidad, la primera causa de fallecimiento a nivel mundial $(70 \%)$.

En ese escenario la evaluación preoperatoria representa un reto, debido a que las complicaciones pulmonares son tan comunes y mórbidas como las complicaciones cardiacas, con una tasa de mortalidad elevada cercana a 50\%, lo cual también lo evidencia los datos reportado por el programa nacional de mejora de la calidad quirúrgica (NSQIP), donde las complicaciones pulmonares posoperatorias obtuvieron un $6 \%(2,3)$. Igualmente los eventos cardiovasculares requieren atención con el objeto de identificar grupos de riesgo, debido a que se ha documentado una incidencia de $3 \%$, como también se referencia al tromboembolismo venoso-TVP (trombosis venosa profunda, embolia pulmonar), constituyendo otra complicación con elevada morbimortalidad que afecta aproximadamente a uno de cada cuatro pacientes quirúrgicos generales intervenidos que no reciben medidas para su prevención (4). La embolia pulmonar es una de las causas prevenibles más comunes de muertes en el hospital después de una cirugía (5).

Ante las consideraciones anteriores, los médicos involucrados en la evaluación preoperatoria tienen como fin detectar factores de riesgo que pueden aumentar las complicaciones de la cirugía por encima del valor inicial y proponer estrategias para reducirlo, para lo cual es necesario estratificar a los pacientes en función de los posibles eventos adversos perioperatorios en relación con las cardiovasculares están descritos los índices de Goldman, Detsky y Lee, en lo que respecta a la neumonía aunque no hay modelos de estratificación de riesgo pulmonar en cirugías generales validados hasta el momento, el American College of Physicians (ACP) adoptó algunas escalas de estimaciones del riesgo de complicaciones respiratorias específica y para la evaluación del riesgo de tromboembolismo venoso (TEV), la escala de Caprini es válida para pacientes quirúrgicos (6-10).
La calculadora de riesgo quirúrgico del Programa Nacional de Mejoramiento de la Calidad Quirúrgica del Colegio Estadounidense de Cirujanos (ACS-NSQIP) es una herramienta interactiva de riesgo publicada por primera vez en 2013 como un software en línea que solicita información sobre datos demográficos, estado funcional, comorbilidades y la clase de clasificación funcional de la Asociación Americana de Anestesiología (ASA) (11).

La importancia de este estudio radica que el Hospital Central del Instituto Venezolano de los Seguros Sociales (IVSS) Dr. Miguel Pérez Carreño, maneja un volumen alto de cirugías, el cual se ha visto limitado ante la crisis socioeconómica que afronta el país, motivo por lo cual consideramos la necesidad de comparar la calculadora ACS NSQIP con respecto a los índices estandarizados para riesgo de neumonía, cardiaco, y tromboembolismo venoso.

\section{Material y métodos \\ Diseño y ubicación del estudio}

Es un estudio de único centro, transversal y correlacional en pacientes mayores de 35 años, sometidos a cirugía no cardiacas, a cargo de los servicios de cirugía general, ginecoobstetricia, urología, cirugía plástica, neurocirugía y traumatología, del Hospital Central del IVSS “Dr. Miguel Pérez Carreño", que asistieron al área de consulta preoperatoria de medicina interna y emergencia de los servicios antes mencionados, durante el periodo comprendido entre junio de 2018 y marzo de 2019. Se excluyeron aquellos pacientes trasladados a otro hospital, con alta hospitalaria, neumonía, TVP o evento cardiovascular al momento de la evaluación.

Se realizó un modelo de registro, donde se diligencia el número de historia clínica de ingreso, antecedentes personales, datos relativos a la intervención quirúrgica, examen físico, datos relativos a su estancia en unidades de recuperación posanestésica, reanimación, unidades de cuidados intensivos, resultados de índices de riesgos preoperatorios utilizados y evaluación dentro de los 30 días posoperatorio. Para mantener la coherencia, la misma persona realizó toda la revisión de la historia clínica, examen físico e ingresó a todos los pacientes en la calculadora de riesgo, asignando a todos el código CPT y estratificó riesgos mediante las escalas de LEE, de neumonía del American College of Physicians ACP) y Caprini según el puntaje. Los resultados detectados posterior a la intervención quirúrgica incluyeron: neumonía, 
tromboembolismo venoso (TEV), y complicaciones cardiovasculares (infarto del miocardio, parada cardiaca, edema de pulmón, arritmias de novo, enfermedad cerebrovascular). En el posoperatorio el seguimiento se realizó a través del servicio de consulta externa del servicio quirúrgico interventor y/o por vía telefónica evaluando la presencia de las morbilidades antes mencionadas hasta por 30 días.

\section{Análisis de los datos}

Las variables continuas se resumieron con medias y desviación estándar si la distribución es normal o en medianas y rangos intercuartílicos si la distribución es no normal; las variables categóricas se resumieron con recuentos de frecuencia y porcentajes. La capacidad de la calculadora para distinguir entre pacientes con o sin las complicaciones correspondientes se resumió utilizando el área bajo una curva de características operativas (AUC) del receptor. El AUC puede variar de 0.5-1.0, con valores más altos que indican capacidad de predicción o discriminación mejoradas. Los análisis estadísticos se realizaron con SPSS 22. Los valores de $\mathrm{p}<0.05$ se consideraron estadísticamente significativos. Los resultados fueron expresados en tablas y figuras. La muestra fue intencional y se utilizó la imputación de datos si el subregistro es menor del $20 \%$ con el fin de mitigar este efecto.

\section{Revisión del comité de ética}

Todos los pacientes firmaron el consentimiento informado que contó con la aprobación del Comité de Bioética del Hospital Central del IVSS Dr. Miguel Pérez Carreño en cumplimiento de lo establecido por la declaración de Helsinki.

\section{Resultados}

\section{Datos epidemiológicos y condiciones médicas de los pacientes con cirugías no cardiacas}

Se seleccionaron 98 pacientes (Figura 1), con una edad promedio de 55 años \pm 14 , donde el sexo femenino representó $54.1 \%(\mathrm{n}=53)$. El 62.2\% $(\mathrm{n}=61)$ de los pacientes tenía un IMC normal. En relación con la distribución del servicio quirúrgico predomino cirugía general con $73.5 \%$ (n =72), así mismo la mayoría de las cirugías fueron electivas con $60.2 \%(\mathrm{n}=59)$. Con respecto a las escalas de riesgo preoperatorio el ASA catalogó al $59.2 \%(\mathrm{n}=72)$ en clase II. El índice de LEE el rango más frecuente fue el II con $67.3 \%(n=66)$. El 53\% $(n=52)$ se estratificaron como muy alto riesgo para enfermedad tromboembólica según la escala Caprini. La escala de riesgo de neumonía de ACP utilizada para evaluar riesgo de complicaciones respiratorias, la clase predominante fue la II con una proporción de $40.8 \%$ ( $\mathrm{n}=$ 40) (Tabla 1).

El 91.8\% ( $\mathrm{n}=90)$ de los pacientes en cuanto al estatus funcional se encontraba en dependientes. Las tres comorbilidades más frecuentes encontradas fueron la hipertensión arterial $36.7 \%(\mathrm{n}=36)$, tabaquismo $17.3 \%(\mathrm{n}=17)$ y diabetes mellitus $10.2 \%(\mathrm{n}=10)$ (Tabla 2$)$.
Tabla 1. Características sociodemográficas de los pacientes quirúrgicos no cardíacos del Hospital del IVSS "Dr. Miguel Pérez Carreño".

\begin{tabular}{|c|c|}
\hline Variables & $\begin{array}{c}\text { Estadísticos } \\
\mathbf{n}(\%)\end{array}$ \\
\hline $\mathbf{N}$ & $98(\%)$ \\
\hline Edad (años) (*) & $55 \pm 14$ \\
\hline \multicolumn{2}{|l|}{ Sexo } \\
\hline Femenino & $53(54.1)$ \\
\hline Masculino & $45(45.9)$ \\
\hline \multicolumn{2}{|l|}{ Índice de masa corporal (IMC) } \\
\hline Bajo peso & $2(2)$ \\
\hline Normal & $61(62.2)$ \\
\hline Sobrepeso & $21(21.4)$ \\
\hline Obesidad & $14(14.3)$ \\
\hline \multicolumn{2}{|l|}{ Servicio quirúrgico } \\
\hline Cirugía general & $72(73.5)$ \\
\hline Traumatología & $16(16.3)$ \\
\hline Neurocirugía & $4(4.1)$ \\
\hline Urología & $4(4.1)$ \\
\hline Ginecoobstetricia & $2(2)$ \\
\hline \multicolumn{2}{|l|}{ Cirugía de emergencia } \\
\hline Sí & $39(39.8)$ \\
\hline No & $59(60.2)$ \\
\hline \multicolumn{2}{|l|}{ Clasificación ASA } \\
\hline I (paciente sano) & $25(255)$ \\
\hline II (enfermedad sistémica leve) & $58(59.2)$ \\
\hline III (enfermedad sistémica grave) & $15(15.3)$ \\
\hline \multicolumn{2}{|l|}{ Índice cardíaco Lee } \\
\hline Clase I & $28(28.6)$ \\
\hline Clase II & $66(67.3)$ \\
\hline Clase III & $4(4.1)$ \\
\hline \multicolumn{2}{|l|}{ Escala de Caprini } \\
\hline Bajo & $6(6.1)$ \\
\hline Moderado & $8(8.2)$ \\
\hline Alto & $32(32.7)$ \\
\hline Muy alto & $52(53.1)$ \\
\hline \multicolumn{2}{|l|}{ Escala ACP } \\
\hline Clase I & $33(33.7)$ \\
\hline Clase II & $40(40.8)$ \\
\hline Clase III & $25(25.5)$ \\
\hline \multicolumn{2}{|c|}{ IVSS: Instituto Venezolano de los Seguros Sociales. } \\
\hline \multicolumn{2}{|c|}{ Escala de American College of Physicians (ACP). } \\
\hline \multicolumn{2}{|l|}{ (*) media \pm desviación estándar. } \\
\hline \multicolumn{2}{|c|}{ Fuente: preparado por los autores a partir de los resultados del estudio. } \\
\hline
\end{tabular}




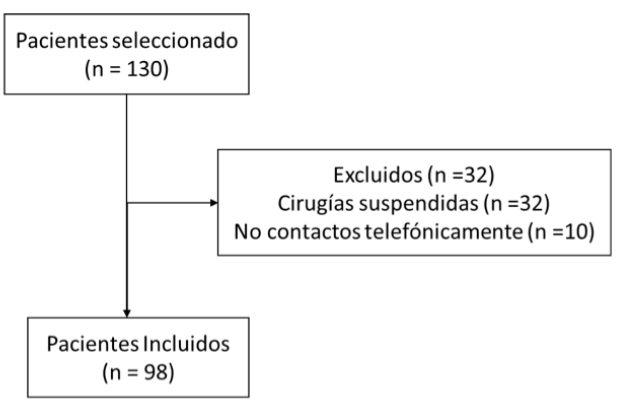

Figura 1. Flujograma de selección de pacientes quirúrgicos no cardiacos del Hospital del IVSS Dr. Miguel Pérez Carreño.

\section{Evaluación de las complicaciones posoperatorias}

De acuerdo con las complicaciones posoperatorias relacionadas con el tipo de cirugía, se documentaron en $23.4 \%$ $(\mathrm{n}=23)$, donde la neumonía fue la más frecuente con $14.2 \%$ $(n=14)$. La mayoría de las complicaciones se presentaron en los pacientes hospitalizados en cirugía general representando el 20.4\% $(n=20)$. En cuanto a las complicaciones posoperatorias según tiempo de presentación se observó que $78.2 \%(\mathrm{n}=18)$ de los casos se presentaron antes de los 15 días, donde la neumonía represento un $50 \%(n=9)$, las cuales estuvieron conexas con el grupo de pacientes que fue intervenido de emergencia con $52.1 \%(\mathrm{n}=12)($ Tabla 3$)$.

\section{Evaluación del rendimiento de la calculadora ACS- NSQIP}

Las curvas de característica operativa del receptor (ROC) se crearon para cada tipo de complicación. En el análisis general, sólo resultaron ser significativos los modelos de curva ROC de clasificación para neumonía (ABC: 0.695 IC 95\% [0.54-0.85], p = 0.017) y complicaciones cardia-
Tabla 2. Condiciones médicas de los pacientes quirúrgicos no cardíacos del Hospital del IVSS “Dr. Miguel Pérez Carreño".

\begin{tabular}{|c|c|c|}
\hline Condiciones médicas & $\mathbf{n}$ & $\%$ \\
\hline \multicolumn{3}{|l|}{ Estatus funcional } \\
\hline No dependiente & 90 & 91.8 \\
\hline Parcialmente dependiente & 7 & 7.1 \\
\hline Dependiente & 1 & 1.0 \\
\hline Hipertensión arterial & 36 & 36.7 \\
\hline Fumador $\ddagger$ & 17 & 17.3 \\
\hline Diabetes mellitus & 10 & 10.2 \\
\hline Disnea & 5 & 5.1 \\
\hline Esteroides* & 2 & 2.0 \\
\hline Ascitis* & 2 & 2.0 \\
\hline Cáncer diseminado & 2 & 2.0 \\
\hline Insuficiencia cardiaca* & 1 & 1.0 \\
\hline EPOC & 1 & 1.0 \\
\hline Falla renal aguda II & 1 & 1.0 \\
\hline \multicolumn{3}{|c|}{ 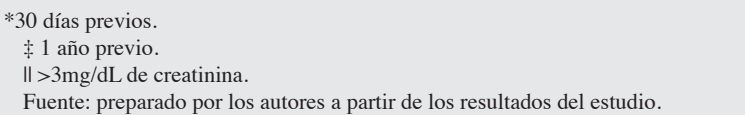 } \\
\hline
\end{tabular}

cas (ABC: 0.739 IC 95\% [0.55-0,93], $\mathrm{p}=0.036)$. En lo que respecta a los estándares LEE y ACP no presentaron significancia estadística en la estimación de riesgo de complicación cardiovascular y neumonía ROC, con valores de ABC: 0.560 IC 95\% [0.37-0.75], $\mathrm{p}=0.601$ y ABC: 0.624 IC $95 \%$ [0.48-0.77], $\mathrm{p}=0.140$ respectivamente (Figuras

Tabla 3. Relación de complicaciones postoperatorias de los pacientes quirúrgicos no cardíacos de acuerdo con el tipo de cirugía y tiempo de presentación.

\begin{tabular}{|c|c|c|c|c|c|c|c|c|}
\hline & \multicolumn{2}{|c|}{ Neumonía } & \multicolumn{2}{|c|}{$\mathrm{CV}$} & \multicolumn{2}{|c|}{ TVP } & \multicolumn{2}{|c|}{ Total } \\
\hline & $\mathrm{n}$ & $\%$ & $\mathrm{n}$ & $\%$ & $\mathrm{n}$ & $\%$ & $\mathrm{n}$ & $\%$ \\
\hline Cirugía & 11 & 55.0 & 7 & 35.0 & 2 & 10.0 & 20 & 100.0 \\
\hline Neurocirugía & 2 & 100 & 0 & 0.0 & 0 & 0.0 & 2 & 100.0 \\
\hline Urología & 1 & 100 & 0 & 0.0 & 0 & 0.0 & 1 & 100.0 \\
\hline Total & 14 & 60.9 & 7 & 30.4 & 2 & 8.7 & 23 & 100.0 \\
\hline Postoperatorio menor a 15 días & 9 & 50.0 & 7 & 38.9 & 2 & 11.1 & 18 & 100.0 \\
\hline Postoperatorio de 15 días o mayor & 5 & 100.0 & 0 & 0.0 & 0 & 0.0 & 5 & 100.0 \\
\hline Total & 14 & 60.9 & 7 & 30.4 & 2 & 8.7 & 23 & 100.0 \\
\hline Casos en base a la población total $(\mathrm{n}=98)$ & 14 & 14.2 & 7 & 7.1 & 2 & 2.0 & 23 & 23.4 \\
\hline
\end{tabular}


2-4). En los gráficos mencionados no se encuentra la curva de características operativas del receptor para el estándar de LEE. De estos, solo el tromboembolismo venoso tenía un intervalo de confianza estrecho, sin embargo, no resultó ser estadísticamente significativo para el ABC.

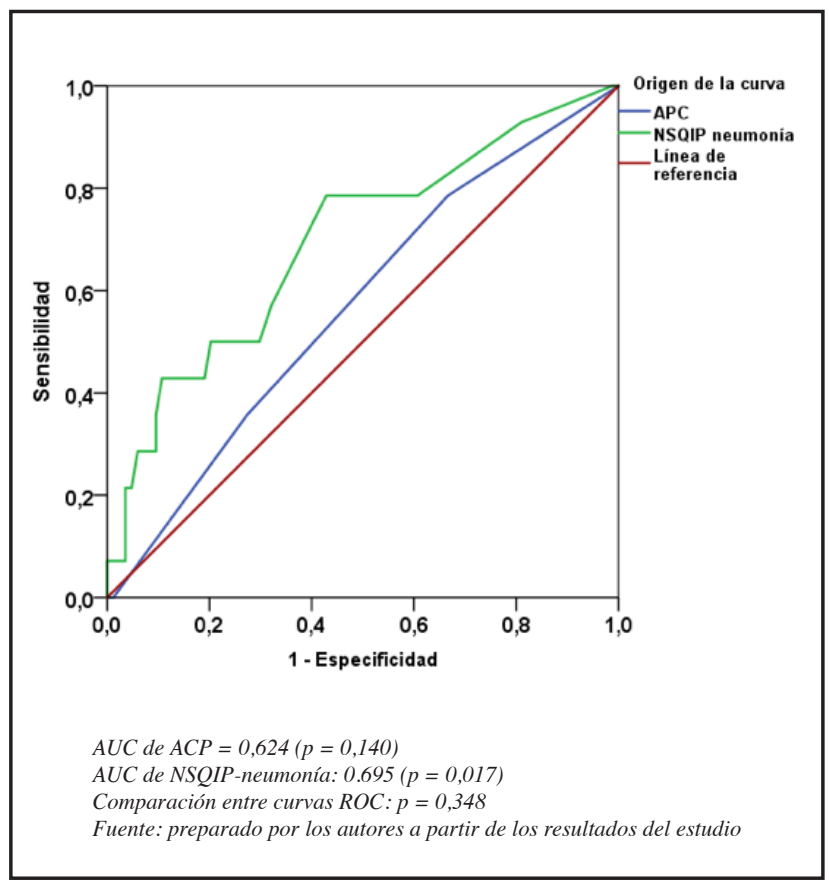

Figura 2. Curvas de característica operativa del receptor (ROC) para NSQIP neumonía y complicaciones de neumonía.

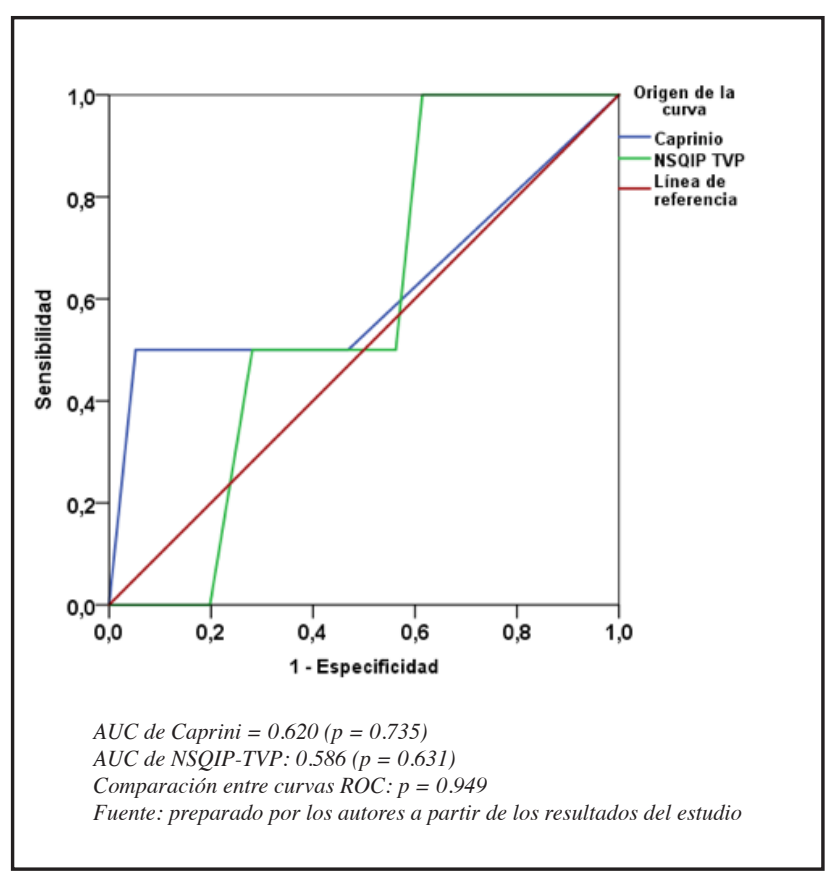

Figura 3. Curvas de característica operativa del receptor (ROC) para NSQIP TVP y complicaciones de TVP.

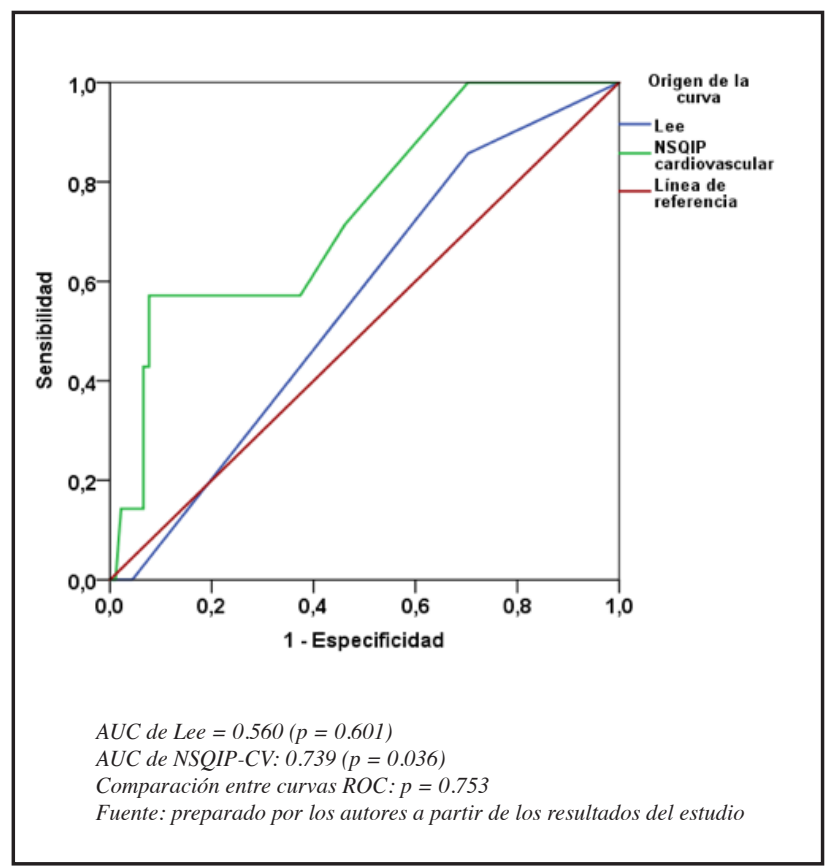

Figura 4. Curvas de característica operativa del receptor (ROC) para NSQIP cardiovascular y complicaciones cardiovasculares.

\section{Discusión}

En este estudio se aplicó la calculadora ACS-NSQIP en comparación con las escalas LEE, Caprini y ACP, como característica de la muestra hubo más representatividad del sexo femenino, ubicado en la sexta década de la vida, encontrando que el servicio quirúrgico preponderante fue el de cirugía general, lo cual está en correspondencia con el estudio (12) realizado en Caracas en el Hospital del IVSS Dr. Domingo Luciani; sin embargo, estos no son coincidentes con respecto a la edad y sexo de los pacientes descrito por Müller y colaboradores (13).

El estudio de Contreras M (14), demostró que la mayoría de sus pacientes se ubicaron en clase I del ASA, igualmente las tres primeras condiciones médicas descritas fueron la dislipidemia, caries y la obesidad, estos hallazgos difieren con los actuales resultados, donde demostramos que la clase II de ASA represento el mayor número de casos, y la hipertensión arterial, el tabaquismo y la diabetes correspondieron a las tres entidades más frecuentes con respecto a las condiciones médicas de los pacientes, estos resultados pueden estar relacionado con el mayor promedio de edad del presente estudio.

La principal complicación posoperatoria obtenida, fue la neumonía con $60.9 \%$, que va en consonancia con el estudio de Gupta S. y colaboradores (15), donde de 59 pacientes con complicaciones respiratorias posoperatorias, $48(81.3 \%)$ presentaron neumonía. Sin embargo, su epidemiología varía ampliamente como lo demuestran Chughtai M. y colaboradores que reportó una incidencia de $0.97 \%$ (13). El estudio de Fernández E. y colaboradores, relaciono que los casos que presentaron complicaciones pulmonares tenían como factores de riesgos el tabaquismo, enfermedad pulmonar obstructiva crónica, diabetes, cirugía de mayor duración, 
hipotensión intraoperatoria, mayor administración de fluidoterapia, y transfusiones, cabe destacar que nuestros pacientes coincidían con la presencia de varios de estos factores. En cuanto a las complicaciones cardiovasculares estuvieron presentes en $7.2 \%$ de la cohorte, hallazgo semejante a la descrita en análisis previos que estimaron su aparición en 5.8\% (16). Por otra parte, los estudios epidemiológicos demuestran que la incidencia de TVP sintomática posoperatoria se sitúa alrededor de $2 \%$ tras intervenciones consideradas de alto riesgo (17), hecho equiparable con el resultado alcanzado.

En cuanto, al rendimiento de la calculadora el análisis encontró asociaciones estadísticas entre las estimaciones de riesgo y la ocurrencia de eventos, para las categorías de complicaciones cardiacas y neumonía, incluso superior a los estándares respectivos índice LEE y ACP, se equipará con lo obtenido en el estudio de Edelstein y colaboradores (18) donde la calculadora ACS-NSQIP, predijo las complicaciones cardiacas $(p<0.001)$ y neumonía $(p<0.001)$. Caso contrario ocurrió con el tromboembolismo venoso donde la escala Caprini fue superior con ROC $0.620(\mathrm{p}=0.735)$ en contraste con la calculadora ROC $0.586(\mathrm{p}=0.631)$ no significativo probablemente asociada al escaso número de eventos, este resultado también es comparable con las conclusiones realizadas por Yap y colaboradores (19) los cuales evidenciaron que la calculadora ACS-NSQIP tenía buena capacidad de predicción para neumonía (AUC 0.93), mortalidad por todas las causas (AUC 0.89) y morbilidad (AUC 0.88); sin embargo para insuficiencia renal, retorno a cirugía, infección del sitio quirúrgico, infección del tracto urinario y tromboembolia venosa su predicción era escasa a regular.

En cuanto a las limitaciones del estudio, podemos mencionar el tamaño de la muestra como resultado de los problemas que padece el sistema de salud venezolano que impacta en este tipo de servicios de salud, lo cual también pudo afectar el bajo volumen de eventos que probablemente comprometió el rendimiento de la calculadora ACS-NSQIP.

\section{Conclusión}

Consideramos que la calculadora (ACS-NSQIP), constituye una herramienta de riesgo de utilidad para predecir complicaciones posoperatorias como neumonía y eventos cardiovasculares. Sin embargo, se hace indispensable la realización de un estudio con mayor número de pacientes que permita detectar más eventos para validar aún más la capacidad de predicción de la calculadora en estas entidades e inclusive en la enfermedad tromboembólica venosa. Como debilidad del presente estudio podemos mencionar que no se realizó el cálculo muestral.

\section{Financiamiento}

El presente trabajo ha sido financiado por el Centro Biomédico de Investigación en Medicina Interna-CEBIMI.

\section{Referencias}

1. Forouzanfar M, Alexander L, Anderson R, Bachman V, Biryukov S, et al. Global, regional, and national comparative risk assessment of 79 behavioural, environmental and occupational, and metabolic risks or clusters of risks in 188 countries, 1990-2013: a systematic analysis for the Global Burden of Disease Study 2013. Lancet. Vol 386, Dec 2015, p2287-2323. doi: 10.1016/S01406736(15)00128-2.

2. Arozullah A, Khuri S, Henderson W, Daley J. Development and Validation of a Multifactorial Risk Index for Predicting Postoperative Pneumonia after Major Noncardiac Surgery. Ann. Intern. Med. 2001; 135(10):847. doi: 10.7326/00034819-135-10-200111200-00005.

3. Yang CK, Teng A, Lee DY, Rose K. Pulmonary complications after major abdominal surgery: National Surgical Quality Improvement Program analysis. $J$ Surg Res. 2015 Oct;198(2):441-9. doi: 10.1016/j.jss.2015.03.028

4. Smilowitz NR, Gupta N, Ramakrishna H, Guo Y, Berger JS. Bangalore S. Perioperative Major Adverse Cardiovascular and Cerebrovascular Events Associated With Noncardiac Surgery. JAMA Cardiol. 2017 Feb 1;2(2):181-187. doi: 10.1001/jamacardio.2016.4792.

5. Lindblad B, Eriksson A, Bergqvist D. Autopsy-verified pulmonary embolism in a surgical department: analysis of the period from 1951 to 1988. Br J Surg 1991; 78:849.

6. Goldman L, Caldera DL, Nussbaum SR, Southwick FS, Krogstad D, Murray B, et al. Multifactorial index of cardiac risk in noncardiac surgical procedures. $N$ Engl J Med. 1977; 297: 845-50.

7. Detsky AS, Abrams HB, Forbath N, Scott JG, Hilliard JR. Cardiac assessment for patients undergoing noncardiac surgery. A multifactorial clinical risk index. Arch Intern Med. 1986; 146: 2131

8. Lee TH, Marcantonio ER, Mangione CM, Thomas EJ, Polanczyk CA, Cook $\mathbf{E F}$, et al. Derivation and prospective validation of a simple index for prediction of cardiac risk of major noncardiac surgery. Circulation. 1999; 100:1043-9

9. Arozullah AM, Khuri SF, Henderson WG, Daley J. Participants in the National Veterans Affairs Surgical Quality Improvement Program. Development and validation of a multifactorial risk index for predicting postoperative pneumonia after major noncardiac surgery. Ann Intern Med. 2001 Nov 20;135(10):847-57. doi: 10.7326/0003-4819-135-10-200111200-00005.

10. Obi AT, Pannucci CJ, Nackashi A, Abdullah N, Alvarez R, Bahl V, et al. Validation of the Caprini Venous Thromboembolism Risk Assessment Model in Critically Ill Surgical Patients. JAMA Surg. 2015 Oct;150(10):941-8. doi: 10.1001/ jamasurg.2015.1841

11. Liu Y, Cohen ME, Hall BL, Ko CY, Bilimoria KY. Evaluation and Enhancement of Calibration in the American College of Surgeons NSQIP Surgical Risk Calculator. J Am Coll Surg. 2016 Aug;223(2):231-9. doi: 10.1016/j.jamcollsurg.2016.03.040

12. Cortázar L, Vera J, Giannoni M. Evaluación preoperatoria y riesgo de mortalidad posoperatoria en cirugía electiva experiencia en el Hospital Domingo Luciani. Rev Venz Med Interna; 32(2): 22-53, 1992.

13. Müller EM, Herrmann E, Schmandra T, Weigel TF, Hanisch E, Buia A. Report of a Quality Improvement Program for Reducing Postoperative Complications by Using a Surgical Risk Calculator in a Cohort of General Surgery Patients. World J Surg. 2020 Jun;44(6):1745-1754. doi: 10.1007/s00268-020-05393-6.

14. Contreras M. Evaluación médica preoperatoria como predictor de riesgo quirúrgico y no quirúrgico. Comunidad y Salud. 2010 Jun; 8(1): 083-096.

15. Gupta S, Fernandes RJ, Rao JS, Dhanpal R. Perioperative risk factors for pulmonary complications after non-cardiac surgery. J Anaesthesiol Clin Pharmacol. 2020 Jan-Mar;36(1):88-93. doi: 10.4103/joacp.JOACP_54_19.

16. Pantoja H, Ramos H, Tovar W. Sensitivity, specificity and predictive values of the Goldman, Detsky and Lee cardiac indices. Rev. Col. Anest. 2014; 42 (3):184191. doi.org/10.1016/j.rca.2014.02.005

17. Wessel N, Gerner T. Thromboembolic complications in ambulatory surgery. A retrospective study of 1691 patients. Tidsskr Nor Laegeforen. 1996; 116:615-6.

18. Edelstein AI, Kwasny MJ, Suleiman LI, Khakhkhar RH, Moore MA, Beal MD, Manning DW. Can the American College of Surgeons Risk Calculator Predict 30-Day Complications After Knee and Hip Arthroplasty? J Arthroplasty. 2015 Sep;30(9 Suppl):5-10. doi: 10.1016/j.arth.2015.01.057.

19. Yap MKC, Ang KF, Gonzales-Porciuncula LA, Esposo E. Validation of the American College of Surgeons Risk Calculator for preoperative risk stratification Heart Asia. 2018 May 17;10(2): e010993. doi: 10.1136/heartasia-2017-010993. 